\title{
Gravitational waves from inspiral into massive black holes
}

\author{
Scott A. Hughes \\ Theoretical Astrophysics, California Institute of Technology, Pasadena, CA 91125
}

\begin{abstract}
Space-based gravitational-wave interferometers such as LISA will be sensitive to the inspiral of stellar mass compact objects into black holes with masses in the range of roughly $10^{5}$ solar masses to (a few) $10^{7}$ solar masses. During the last year of inspiral, the compact body spends several hundred thousand orbits spiraling from several Schwarzschild radii to the last stable orbit. The gravitational waves emitted from these orbits probe the strong-field region of the black hole spacetime and can make possible high precision tests and measurements of the black hole's properties. Measuring such waves will require a good theoretical understanding of the waves' properties, which in turn requires a good understanding of strong-field radiation reaction and of properties of the black hole's astrophysical environment which could complicate waveform generation. In these proceedings, I review estimates of the rate at which such inspirals occur in the universe, and discuss what is being done and what must be done further in order to calculate the inspiral waveform.
\end{abstract}

One of the most exciting sources that should be measured by the space-based gravitational-wave interferometer LISA (the Laser Interferometer Space Antenna [1]) is the inspiral of a "small" $\left(1-10 M_{\odot}\right)$ compact body into a massive $\left(10^{5-7} M_{\odot}\right)$ black hole. Such massive black holes reside at the cores of galaxies; the smaller compact bodies will become bound to the hole and spiral into them after undergoing interactions with stars and other objects in the environment of the galactic center. The measurement of gravitational waves from such inspirals will make possible very high precision tests of general relativity, and probe the nature of the galactic core's environment.

To set the stage for understanding why these inspirals are such interesting objects, consider the following estimates: the orbital energy of a small body in an equatorial, prograde orbit of a Kerr black hole is

$$
E^{\mathrm{orb}}=\mu \frac{1-2 v^{2}+q v^{3}}{\sqrt{1-3 v^{2}+2 q v^{3}}}
$$

where $v \equiv \sqrt{M / r}$ and $q \equiv a / M$. (I use units in which $G=c=1$ throughout.) The orbital frequency of the small body is

CP523, Gravitational Waves: Third Edoardo Amaldi Conference, edited by S. Meshkov (C) 2000 American Institute of Physics 1-56396-944-0/00/\$17.00 


$$
\Omega=\frac{M^{1 / 2}}{r^{3 / 2}+a M^{1 / 2}} .
$$

Radiation reaction carries orbital energy away from the system, causing the orbit to shrink. Eventually it shrinks enough that the body reaches the innermost stable circular orbit (ISCO). Orbits inside this radius are dynamically unstable; further radiative evolution tends to push the body into the black hole.

Post-Newtonian theory allows us estimate the rate at which the system loses energy as a power series in the quantity $u \equiv(M \Omega)^{1 / 3}$ (which is roughly the orbital speed) and the black hole's spin $a$. Reference [2] gives the energy loss in such a post-Newtonian expansion:

$$
\frac{d E}{d t}=-\frac{32}{5}\left(\frac{\mu}{M}\right)^{2} u^{10}\left[f_{\mathrm{Schw} .}(u)+f_{\mathrm{spin}}(a, u)\right] .
$$

The prefactor $-32 / 5(\mu / M)^{2} u^{10}$ is the result one gets applying the quadrupole formula to a binary described with Newtonian gravity; the function $f_{\mathrm{Schw}}(u)$ is a (rather high-order) correction appropriate for zero-spin black holes, and $f_{\text {spin }}(a, u)$ is a correction incorporating information about the hole's spin. (Note that this formula is only appropriate for $\mu \ll M$ : it does not incorporate any finite mass ratio corrections.)

Equations (1)-(3) can be used to estimate the time it takes for a small body to spiral from radius $r_{1}$ to $r_{2}$, and the number of gravitational-wave cycles it emits in that time:

$$
\begin{aligned}
T & =\int_{t_{1}}^{t_{2}} d t=\int_{r_{1}}^{r_{2}} \frac{d t}{d r} d r=\int_{r_{1}}^{r_{2}} \frac{d E / d r}{d E / d t} d r \\
N_{\mathrm{cyc}} & =\int_{t_{1}}^{t_{2}} f_{\mathrm{gw}} d t=\int_{r_{1}}^{r_{2}} \frac{\Omega}{\pi} \frac{d t}{d r} d r=\int_{r_{1}}^{r_{2}} \frac{\Omega}{\pi} \frac{d E / d r}{d E / d t} d r .
\end{aligned}
$$

(On the last line, I have assumed that the bulk of the radiation comes out in the quadrupole $m=2$ mode, so that $f_{\mathrm{gw}}=2 \Omega / 2 \pi$.) Consider now the inspiral of a $5 M_{\odot}$ body into a rapidly spinning $(a \simeq M) 10^{6} M_{\odot}$ black hole. The small body spirals from a radius of $8 M$ (in Boyer-Lindquist coordinates) to the ISCO in one year, emitting around $5 \times 10^{5}$ gravitational-wave cycles as it does so. The gravitationalwaves that it emits lie in the frequency band $3 \times 10^{-3} \mathrm{~Hz} \lesssim f \lesssim 3 \times 10^{-2} \mathrm{~Hz}$ - the band to which LISA is most sensitive. Indeed, careful analyses of the detectability of the signal by LISA [3] indicate that such an inspiral should be detected out to a distance of roughly 1 Gigaparsec with amplitude signal-to-noise ratio of around 10 to 100 (depending on factors such as the mass of the small body, the mass of the black hole, and the black hole's spin). The fact that such a large number of cycles are emitted indicates that details of the waveform can in principle be measured to very high precision. Detection of extreme mass-ratio inspirals by LISA offers the possibility of very high precision measurements of the characteristics of extreme strong-field regions of spacetime. 
The high precision tests that LISA will be able to make should allow us to directly map the characteristics of the massive body's spacetime metric and confirm that they in fact exhibit the Kerr metric. Most likely, the way that this will be done will be to measure the multipole moments of the massive body. Fintan Ryan [4] has shown that the gravitational waves which are emitted as a small body spirals into a massive compact object contain a "map" of the massive body's spacetime. By measuring the gravitational waves and decoding the map, one learns the mass and current multipole moments which characterize the massive body. All multipole moments of Kerr black holes are parameterized by the holes' mass $M$ and spin $a$ :

$$
M_{l}+i S_{l}=M(i a)^{l} .
$$

For Kerr black holes, knowledge of the moments $M_{0} \equiv M$ and $S_{1} \equiv a M$ determines all higher moments. This is one way of stating the "no-hair" theorem: The macroscopic properties of a black hole are entirely determined by its mass and spin. (I neglect the astrophysically uninteresting possibility of charged holes.) By measuring gravitational waves from extreme mass ratio inspiral and thereby mapping the massive body's spacetime, LISA will test the no-hair theorem for black holes, determining whether the massive body has multipole moments characteristic of the Kerr metric, or whether the body is something more exotic, such as a boson star.

The waves emitted by extreme mass ratio inspiral are thus likely to be directly measureable by LISA, and are likely to be extremely interesting. One might next wonder whether they occur often enough to be interesting. This question has been examined in some detail by Martin Rees and Steinn Sigurdsson [5,6]. They consider the scatter of stellar mass black holes in the central density cusp of galaxies into tightly bound orbits of the galaxies' central black hole. Occasionally, such a scattering event will put the stellar mass hole into an orbit which is so tightly bound that its future dynamics are driven by gravitational-wave emission, and it becomes an interesting source for LISA. They find that the rate of such events is likely to lie in the range

$$
\frac{1 \text { event }}{\text { year } \mathrm{Gpc}^{3}} \lesssim \mathcal{R} \lesssim \frac{1 \text { event }}{\text { month } \mathrm{Gpc}^{3}} .
$$

Obviously, there are large uncertainties in this calculation. The low mass end of the massive black hole population (which is most relevant to LISA observations) is not as well constrained as the population of very massive black holes $\left(M \gtrsim 10^{8} M_{\odot}\right)$, and there are uncertainties in the rate at which stellar mass black holes are "fed" into the central hole to produce extreme mass ratio binaries. However, the lower end of the rate estimate (7) is based on very conservative estimates. We may rather robustly estimate that the rate measured by LISA will be several events per year out to a Gigaparsec [7].

The waves that LISA will measure from these sources will come from orbits that are rather eccentric and inclined with respect to the black hole's equatorial plane [8]. To best interpret the measured waves (and, indeed, in order to improve 
the odds of seeing the waves at all in the detector's noisy data stream), it will be necessary to have some theoretical modeling of the orbit and the waves that it emits as gravitational radiation reduces the orbit's energy and angular momentum. We expect that the radius, inclination angle, and eccentricity of the orbit will change as radiative backreaction drives the system's evolution. Some means of understanding these changes, in detail, is needed in order to model the waves' evolution accurately.

Before discussing work in radiation reaction, it is necessary to take a sanity check. Relativity theorists often work in a very idealized universe: their extreme mass ratio binary is likely to consist of a big black hole, a small body, and gravitational waves. In the real astrophysical world, there will be complications to this pretty (but highly idealized) picture. One should worry whether the complications render the relativity theorist's modeling invalid.

Perhaps the most important such complication arises from interaction between the small inspiraling body and material accreting onto the massive black hole. Recently, Narayan has analyzed this interaction and concluded that, in almost all cases, accretion induced drag is unlikely to significantly influence extreme mass ratio inspiral [9]. This conclusion is based on the fact that in the majority of cases, the rate at which the central black hole accretes gas from its environment is rather low (several orders of magnitude less than the Eddington rate [10]). For these "normal" galaxies, much evidence $[9,10]$ suggests that the gas accretes via an advection dominated accretion flow (ADAF). Narayan's calculation [9] shows that the timescale for ADAF drag to change the orbit's characteristics (e.g., the orbital angular momentum) is many (9 to 16) orders of magnitude longer than the timescale for radiation reaction to change the orbit's characteristics. Thus, the relativity theorist's idealized view of an extreme mass ratio binary is probably quite accurate: radiation reaction is likely the most important factor driving the evolution of extreme mass ratio binaries.

When the mass ratio is extreme, one can analyze the spacetime of the binary using a perturbative expansion: the spacetime metric can be written as a "background" from the central object (which I will assume from now on is a Kerr black hole), plus a perturbation due to the inspiraling body:

$$
g_{\alpha \beta}=g_{\alpha \beta}^{\mathrm{Kerr}}(M, a)+h_{\alpha \beta}(\mu) .
$$

The evolution of the perturbation $h_{\alpha \beta}(\mu)$ should then describe the dynamical evolution of the system. To linear order in the mass ratio $\mu / M$ (which should be adequate for extreme mass ratios), this evolution can be described using perturbation techniques, such as the Teukolsky equation ${ }^{1}$ [11].

When the mass ratio is extreme, the effects of radiation reaction are gentle enough that the system's evolution is adiabatic: the radiation reaction timescale is much longer than the orbital timescale. At any given moment, the trajectory of the small body is very nearly a geodesic, parameterized by the three constants of Kerr

1) The Teukolsky equation actually describes the evolution of a curvature quantity related to the perturbation. 
orbital motion: the energy $E$, the ( $z$-component of) angular momentum $L_{z}$, and the "Carter constant" $Q$. Gravitational-wave emission causes these three constants to change on the radiation reaction timescale. In this adiabatic limit, the evolution of the system can be understood in terms of the evolution of the quantities $\left(E, L_{z}, Q\right)$.

It is well known that gravitational waves carry energy and angular momentum. One might think that they carry "Carter constant" as well, and that therefore one might be able to deduce the effects of radiation reaction by measuring the flux of radiation at infinity and down the event horizon. By measuring the amount of $E$, $L_{z}$, and $Q$ carried in the flux one should be able to deduce how much $E, L_{z}$, and $Q$ are lost from the orbit. This would then allow one to figure out orbits of Kerr black holes radiatively evolve.

This approach does not work. One can deduce the change in the orbit's $E$ and $L_{z}$ by examining radiation fluxes, but one cannot so deduce the change in $Q$ :

$$
\begin{aligned}
\delta E_{\text {orbit }} & =-\delta E_{\text {radiated }}, \\
\delta L_{z, \text { orbit }} & =-\delta L_{z, \text { radiated }} \\
\delta Q_{\text {orbit }} & \neq-\delta Q_{\text {radiated }}
\end{aligned}
$$

The change $\delta Q$ turns out to depend explicitly on the local radiation reaction force, $f^{\mu}=d p^{\mu} / d \tau$, which the small body experiences due to radiative backreaction (see [14]). The properties of this force (and programs to calculate it) are described elsewhere in this volume [12]. Here, it is sufficient to note that an understanding of the radiation reaction force for gravitational radiation reaction lies some time in the future, so that we cannot yet evolve generic Kerr black hole orbits.

There are special cases where evolution of the Carter constant is not such a nasty impediment. One case is the evolution of equatorial orbits. Equatorial orbits have $Q=0$; and, one can easily show that an orbit which starts off equatorial remains equatorial. In this case, one need only evolve the energy and angular momentum. The local radiation reaction force is not needed in this case. Another case is the evolution of circular, non-equatorial orbits. (For non-zero spin, "circular" means "constant Boyer-Lindquist coordinate radius".) Such orbits have recently been shown to remain circular under adiabatic radiation reaction [13]. Thus, in an adiabatic evolution, a system which is initially circular and inclined will remain circular and inclined: the system evolves through a sequence of orbits changing only its radius and inclination angle. By imposing "circular goes to circular", one can write down a simple rule relating the change of the Carter constant to the flux of energy and angular momentum: $\dot{Q}=\dot{Q}\left(\dot{E}, L_{z}\right)$.

Recently, I have examined the evolution of these circular orbits under adiabatic radiation reaction, using a flux-measuring formalism based on the Teukolsky equation. The formalism and results are presented at length in [14]. Some highlights of the results are presented in Figure 1.

Consider the left panel of Figure 1. The horizontal axis is orbital radius $r$; the vertical axis is inclination angle $\iota$. This figure shows the direction, in $(r, \iota)$ phase space, in which radiation reaction tends to push the orbit. This particular analysis 

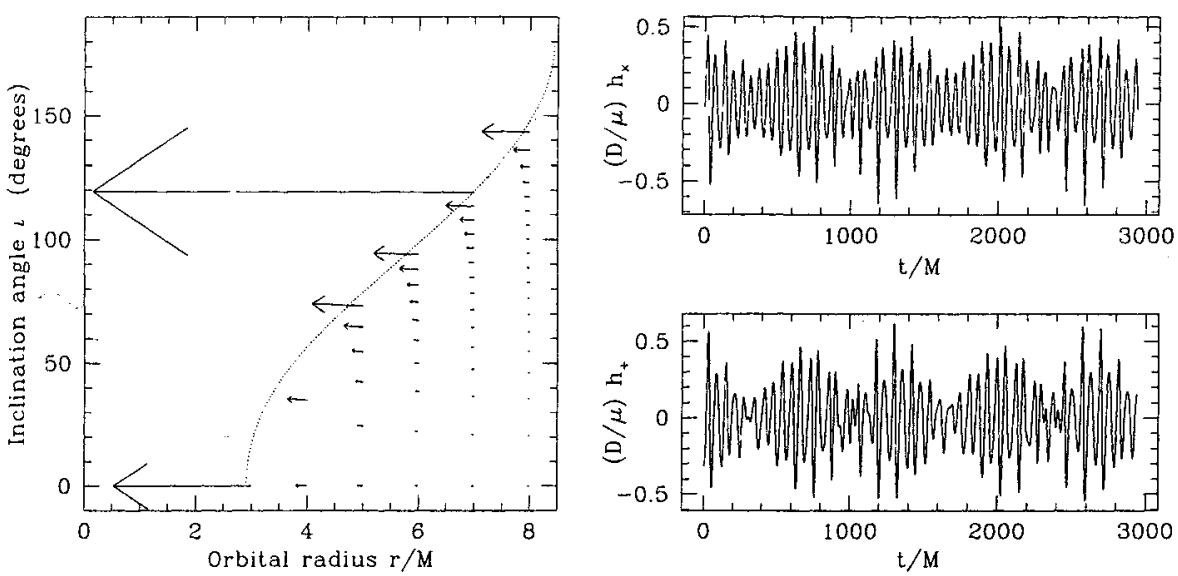

FIGURE 1. The left panel illustrates the effect of radiation reaction on circular, inclined orbits. It is for a black hole with $a=0.8 \mathrm{M}$. The tail of each arrow represents a particular orbit with radius $r$ and inclination angle $\iota$. The arrow indicates the direction in which radiation reaction drives the orbit; the size of the arrow indicates how quickly it is so driven. The right panel is a snapshot of the gravitational waveform emitted during an inspiral into a hole with $a=0.95 \mathrm{M}$. At this moment, the small body is spiraling through $r=7 M, \iota=62.43^{\circ}$.

is for a black hole with $a=0.8 M$. Notice that the orbits are in the strong-field of the hole; the dotted line indicates the maximum inclination angle which the orbit can have and remain stable. Orbits tilted beyond this line are dynamically unstable to small perturbations and plunge into the hole. The tail of each arrow represents a particular orbit. The direction of the arrow gives the direction in which that orbit tends to evolve due to gravitational-wave emission; the arrow's length indicates the relative rate of evolution. In all cases, the direction of the arrow is such that the inclination angle increases: radiation reaction tends to make tilted orbits more inclined. This is exactly what one would have predicted by extrapolating from post-Newtonian theory [15]. The rate at which this inclination angle increases is rather slow - the aspect of the arrows in Figure 1 is nearly flat. Indeed, the value of $d \iota / d t$ in this strong-field regime is roughly 3 times smaller than what postNewtonian theory predicts ${ }^{2}$. An interesting feature of Figure 1 is the very long arrow at $r=7 M, \iota \simeq 120^{\circ}$. This orbit lies extremely close to the marginally stable orbit: it is at $\iota=119.194^{\circ}$; the marginally stable orbit is at $\iota=119.670^{\circ}$. This orbit is barely dynamically stable, so a small push has drastic effects.

The right panel of Figure 1 shows a portion of the gravitational waveform emitted during inspiral. The central black hole in this case has $a=0.95 \mathrm{M}$; it lies at

2) Recent analyses are showing that in the extreme strong-field of rapidly rotating holes, the inclination angle changes rather more dramatically, and in the opposite direction: radiation reaction tends to decrease the inclination angle. This work is in progress [16]. 
luminosity distance $D$ from the detector. The waveform here is shown as the small body passes through $r=7 M, \iota=62.43^{\circ}$. Note the low-frequency modulation of both polarizations. This is due to the frame dragging induced precession of the orbital plane - Lense-Thirring precession. Note also the many sharp, shorttimescale features present in the two polarizations. When the spin is high, many harmonics of the small body's fundamental orbital frequencies contribute to the gravitational waveform. This leads to a rather complicated structure; the energy spectrum corresponding to these waveforms extends to rather high frequencies. Accurate measurement of such complicated waveforms will be quite a challenge. However, the payoff is likely to be immense.

I thank Kip Thorne and Steinn Sigurdsson for help and advice in writing this talk; I also thank Ramesh Narayan for allowing me to use a preliminary draft of Ref. [9]. I am indebted to many people who helped me construct my radiation reaction code, including (but not limited to) Sam Finn, Daniel Kennefick, Yuri Levin, Amos Ori, Sterl Phinney, and "The Capra Gang": Lior Burko, Patrick Brady, Éanna Flanagan, Eric Poisson, and Alan Wiseman. This research was support by NSF Grants AST-9731698 and AST-9618537, and NASA Grants NAG5-6840 and NAG57034.

\section{REFERENCES}

1. K. Danzmann et al., LISA - Proposal for a Laser-Interferometric Gravitational Wave Detector in Space, Max-Planck-Institut für Quantenoptik, Report MPQ 233 (1998).

2. Y. Mino, M. Sasaki, M. Shibata, H. Tagoshi, and T. Tanaka, Prog. Theor. Phys. Supplement No. 128 (1997).

3. L. S. Finn and K. S. Thorne, in preparation.

4. F. D. Ryan, Phys. Rev. D 56, 1845 (1997).

5. S. Sigurdsson and M. J. Rees, Mon. Not. R. Astron. Soc. 284, 318 (1997).

6. S. Sigurdsson, Class. Quantum Grav. 14, 1425 (1997).

7. S. Sigurdsson, private communication.

8. D. Hils and P. L. Bender, Astrophys. J. 447, L7 (1995).

9. R. Narayan, Astrophys. J. submitted; also astro-ph/9907328.

10. R. Narayan, R. Mahadevan, and E. Quataert, in Theory of Black Hole Accretion Disks, edited by M. A. Abramowicz, G. Bjornsson, and J. E. Pringle (Cambridge University Press, Cambridge, 1998).

11. S. A. Teukolsky, Astrophys. J. 185, 635 (1973).

12. Y. Mino, these proceedings; L. Burko, these proceedings.

13. D. Kennefick and A. Ori, Phys. Rev. D 53, 4319 (1996); F. D. Ryan, Phys. Rev. D 53, 3064 (1996); Y. Mino, unpublished Ph.D. thesis, Kyoto University (1996).

14. S. A. Hughes, Phys. Rev. D, in press; also gr-qc/9910091.

15. F. D. Ryan, Phys. Rev. D 52, R3159 (1995).

16. S. A. Hughes, in preparation. 